\title{
ABORDAGEM DA DOENÇA CAROTÍDEA NA FASE AGUDA DO ACIDENTE VASCULAR CEREBRAL
}

\author{
Opinião nacional
}

\author{
Rubens José Gagliardi, Charles André, Marcia Maiumi Fukujima, \\ Sebastião Eurico Melo-Souza, Viviane Flumignan Zétola*
}

\begin{abstract}
RESUMO - Com finalidade de orientar e oferecer subsídios para a conduta diagnóstica e terapêutica em diferentes situações dentro das doenças cerebrovasculares, a Sociedade Brasileira de Doenças Cerebrovascularesconstituiu um comitê composto por especialistas de diferentes áreas do Brasil, que emitiram um parecer, denominado "Opinião Nacional", redigido nos moldes dos consensos. O presente artigo analisa a "Doença Carotídea na Fase Aguda do AVC", abordando separadamente os seguintes tópicos: métodos de deteç̧ão de estenose carotídea na fase aguda; quando e como intervir sobre a carótida na fase aguda; tratamento clínico e marcadores de inflamação. São apresentados os comentários, baseados na literatura e na opinião pessoal dos participantes, e é descrito, como conclusão em cada item, a opinião do grupo.
\end{abstract}

PALAVRAS-CHAVE: doença carotídea, estenose, marcadores de inflamação.

\begin{abstract}
Management of carotid disease in acute phase of stroke: national opinion
ABSTRACT - The Brazilian Stroke Society constituted a committee composed by specialists from different areas of Brazil that emitted a viewpoint called "National Opinion", in a consensus pattern. The study purpose is to guide and offer subsidies for diagnosis and therapeutical plans for different situations in cerebrovascular disease The present article analyzes "carotid disease in the acute phase of stroke", approaching separately the following topics: methods for detection of carotid stenosis in acute phase; when and how intervene on the carotid during the acute phase; clinical treatment and inflammatory markers. Comments based on literature and participants personal opinion are described, and conclusion are the group opinion.
\end{abstract}

KEY WORDS: carotid disease, stenosis, Brazil, inflammation markers.

O comprometimento direto das artérias carótidas por vários mecanismos - em especial aterotrombose, embolia cardíaca ou arterial, dissecção - é bastante comum como causa de infarto cerebral. Além disso, a doença obstrutiva grave da carótida pode contribuir para o agravamento das lesões isquêmicas por outros mecanismos. Com finalidade de orientar e oferecer subsídios para a conduta diagnóstica e terapêutica nesta e em outras situações comuns, a Sociedade Brasileira de Doenças Cerebrovasculares constituiu um comitê composto por especialistas de diferentes áreas do Brasil, que emitiram um parecer, denominado "Opinião Nacional" **

Foram analisados separadamente 4 itens que $m$ e recem destaque dentro do tópico "Doença Carotídea". As conclusões foram embasadas em ampla revisão das evidências disponíveis na literatura pertinente, levando em conta a experiência pessoal dos participantes e as particularidades - como a disponibilidade de métodos - existentes em nosso meio. Sempre que possível, as orientações foram classificadas em níveis de evidência. Para tal, foram utilizados, no presente estudo, critérios baseados nas recomendações seguidas pela EUSI "European Stroke Iniciative"1 (Tabela).

\section{MÉTODOS DE DETECÇÃO DE ESTENOSE CAROTÍDEA NA FASE AGUDA}

Diante de um caso suspeito de doença caro tí-

\footnotetext{
*Pela Sociedade Brasileira de Doenças Cerebrovasculares; **O fórum de "Opinião Nacional" foi realizado em São Paulo-SP, no dia $1^{\circ}$ de julho de 2004. Os temas de trabalho foram divididos para análise inicial por grupos de 5 a 6 neurologistas. Um esboço de texto foi proposto para análise em plenário em leitura pública. Comentários, críticas e sugestões de mudanças foram amplamente debatidos por este plenário e incorporados ao texto final, redigido pelo grupo responsável pelo tema.
}

Recebido 3 Novembro 2004, recebido na forma final 11 Fevereiro 2005. Aceito 5 Abril 2005. 
Tabela. Níveis de evidência: terapia, prevenção e risco.

1. Ensaio clínico randomizado (ECR) ou revisão sistemática (RS) de ECRs com desfechos clínicos

2. ECR ou RS de ECR de menor qualidade: Com desfechos substitutos validados

Com análise de subgrupos ou de hipóteses a posteriori Com desfechos clínicos, mas de menor rigor metodológico

3. ECR com desfechos substitutos não-validados Estudo de caso-controle

4. Estudo com desfecho clínico, mas com maior potencial de viés (tal como experimento não-comparado e demais estudos observacionais)

5. Fó rum re presentativo ou opinião de especialista sem evidências dos níveis supracitados

Adaptado dos critérios adotados pela EUSI ${ }^{1}$

dea, deve sempre ser recomendada investigação por métodos de imagem e fluxo. Os exames não invasivos e semi-invasivos que podem ser utilizados para a detecção de estenose e oclusão carotídea são:

- EcoDoppler extracraniano (Ultra-sonografia com Doppler ou Duplex-scan): este exame possibilita o reconhecimento do padrão morfológico da placa, e em certa medida uma estimativa de sua composição. Estas características provavelmente influenciam o risco futuro de embolização. Além disso, as estimativas de velocidade de fluxo auxiliam na quantificação funcional da estenose. $O$ método apresenta, entretanto, mesmo em centros especializados, $1-14 \%$ de resultados falso-positivos para estenose grave ( $>70 \%$ de estenose carotídea) (nível 1); são indicados estudos de confiabilidade em cada centro que utilize rotineiramente o método ${ }^{3}$.

- Angiografia por ressonância magnética (Angio-RM): pode ter valor preditivo maior que o $D u$ plex-scan ${ }^{4}$, mas estudo brasileiro indica concordância superior do Duplex-scan com a arteriografia digital'5; ambos os métodos têm confiabilidade baixa para oclusão carotídea ${ }^{3}$ (nível 1). Sugere-se o emprego de aparelhos de 1,5 Tesla.

- Angiografia por tomografia (Angio-TC): estudos de sensibilidade e especificidade sugerem boa correlação com arteriografia digital ${ }^{6}$. O método, principalmente com aparelhos com multidetecção helicoidal, tem vantagens potenciais, como discriminação de características de placa eventualmente importantes para determinar condutas terapêuticas. Foram descritos, entretanto, casos em que as alterações obstrutivas detectadas à angio-TC não puderam ser encontradas em arteriogramas subseqüentes 7,8 (nível 4). Lembrar dos riscos inerentes à administração de contraste iodado.

- Doppler transcraniano: o método tem por objetivo avaliar as alterações hemodinâmicas da estenose carotídea. É exame que não avalia, porém, as características funcionais e morfológicas da placa carotídea. Não deve, assim, ser indicado como único exame complementar na avaliação inicial, devendo ser usado em conjunto com o Duplex-scan extracraniano. A técnica possibilita o reconhecimento do padrão da circulação colateral (nível 2) e o reconhecimento de sinais embólicos originando-se do coração ou das artérias proximais (nível 3). Determina a presença de estenose/oclusão dos principais vasos intracranianos, com maior sensibilidade, especificidade, valor preditivo positivo e negativo na circulação anterior (nível 2) do que na ci rculação posterior (nível 3) ) $^{9}$ Exibe taxas de $94 \%$ de sensibilidade e de $97 \%$ de especificidade para estenose carotídea extracraniana grave proximal (até o sifão carotídeo); e de $81 \%$ de sensibilidade e $96 \%$ de especificidade para estenose distal (póssifão) ${ }^{10}$.

- Arteriografia digital é considerada o padrãoouro para avaliar o grau de estenose arterial, a circulação arterial colateral, a presença de estenose intracraniana associada e de outras lesões assintomáticas potencialmente importantes na determinação da conduta terapêutica"11 (nível 1). De custos elevados, tem também riscos inerentes a sua natureza invasiva - complicações neurológicas maiores, como déficit neurológico persistente, em $0,5 \%$ para acidente vascular cerebral (AVC) e 0,4\% para ataque isquêmico transitório (AIT) (podendo chegar a $4 \%$ em pacientes com alto risco de aterosclerose carotídea); e complicações menores como déficit neurológico reversível em até 24 horas em 4\% dos casos (outras complicações, como hematomas e reações ao contraste, são também relatadas) ${ }^{12,13}$. Atualmente estes riscos são muito baixos em função dos novos materiais hidrofílicos e de novos meios de contraste iso-osmolares. Estes fatores limitam sua indicação rotineira ${ }^{14}$.

Comentários: Há disponibilidade variável de exames não invasivos ou semi-invasivos nos diversos centros hospitalares do país. Também há poucos estudos de confiabilidade e padronização destes exames em nosso meio. Na ausência de estudos de confiabilidade e de impacto financeiro destes métodos, sugere-se o emprego rotineiro e inicial 
de exames não invasivos. Dependendo da indicação clínica, da confiabilidade do método e da disponibilidade local, podem ser indicados outros p rocedimentos diagnósticos, como a angiografia digital arterial.

\section{QUANDO E COMO INTERVIR SOBRE A CARÓTIDA NA FASE AGUDA?}

Existe pouca informação sobre endarterectomia na fase aguda do infarto cerebral (IC), sendo que a maioria dos trabalhos consiste em relatos de casos e pequenas séries. Tais estudos sugerem viabilidade da endart e rectomia e trombectomia de carótidas em situações especiais ${ }^{15,16}$ (nível 4). A endarte rectomia é considerada de risco nessa fase pelo risco aumentado de desenvolvimento de edema cerebral e hiperemia (síndrome de hiperfluxo), principalmente em infartos lobares ${ }^{17}$ (nível 4). Estudos em andamento avaliam criticamente a intervenção ultraprecoce - primeiras 8 horas após o icto por estenose crítica ou oclusão carotídea ${ }^{18}$. O mesmo grau de incerteza cerca as intervenções endovasculares - angioplastia e colocação de stents - na fase aguda, havendo relatos de casos e descrição de pequenas séries sobre o assunto (nível 4).

Parece haver maior benefício e segurança em pacientes com ataque isquêmico transitório (AIT); e também após acidente vascular cerebral (AVC) não incapacitante a partir de 2 semanas após o evento ${ }^{1920}$ (nível 1). Nesta fase, deve-se considerar a indicação precocemente, uma vez que o risco de desenvolver AVC após 90 dias do AIT é alto - $11 \%$, e se concentra $(85 \%)$ nos primeiros 30 dias $^{21}$.

Comentários: A endart e rectomia pode ser indicada precocemente após o AIT - excluir, quando possível, IC por métodos mais sensíveis como a Ressonância Magnética por difusão (DWI/ADC) - e após 2 semanas de AVC não grave ou incapacitante em pacientes clinicamente estáveis (nível 1). Nã o há critérios universalmente aceitos para nortear as decisões sobre intervenção cirúrgica ou tratamento endovascular na fase aguda do IC relacionado a doença carotídea (nível 4). Intervenções são eventualmente cogitadas nas seguintes situações clínicas: estenose crítica, déficit neurológico crescente em que se suspeita de trombose em evolução, AIT repetitivos apesar de tratamento clínico ótimo, dissecção arterial. Pode-se considerar angioplastia precocemente em casos selecionados, como suboclusão arterial, em associação ou não com trombólise intra-arterial (nível 4).

\section{TRATAMENTO CLÍNICO}

Frente à suspeita de AVC agudo relacionado a estenose ou oclusão carotídea, recomenda-se a utilização ultraprecoce de rt-PA venoso (nível 1) e de ácido acetil-salicílico (após 24 horas do uso de t rombolíticos ou imediatamente nos casos em que o trombolítico não for utilizado (nível 1), mantendo-se as recomendações dos consensos brasileiros anteriormente publicados ${ }^{22,23}$.

Os agentes inibidores da enzima de conversão de angiotensina (IECA) encontram hoje indicação estabelecida na prevenção do AVC isquêmico após a fase aguda ${ }^{24,25}$ (nível 1). As estatinas (agentes hipolipemiantes) têm sido amplamente utilizadas para prevenção primária e secundária do AVC, com estudo específico avaliando sua eficácia sendo aguardado para $2006^{26}$ (nível 2 para indicação após o AVC).

Quanto à dissecção carotídea, recente metaanálise não conseguiu demonstrar diferenças nos riscos de morte ou de sobrevivência com déficit em pacientes tratados com anticoagulantes ou com anti-agregantes plaquetários ${ }^{27}$ (nível 2).

Comentários: Recomenda-se a introdução rotineira e precoce de anti-agregantes plaquetários (nível 1). Há tendência a se utilizar também a terapia com IECA (nível 1) ou antagonistas de receptor de angiotensina II (nível 4). Até que haja re spostas mais definitivas sobre o uso de estatinas, pode-se indicar estas drogas, considerando os estudos de prevenção em pacientes coronarianos e outros grupos de alto risco vascular (nível 2). A introdução destas drogas antes da alta hospitalar pode aumentar a aderência ao tratamento.

Não se recomenda anticoagulação rotineira dos pacientes com IC. Pode-se, entretanto, indicar anticoagulação plena parenteral - com heparina nãofracionada e controle estrito de TTPA - em casos específicos e em decisões altamente individualizadas, como ataques isquêmicos recomentes e freqüentes (excluir mecanismos hemodinâmicos para as oscilações sintomáticas), estenose crítica sintomática (por exemplo, até a endarterectomia), déficit progressivo na ausência de melhor explicação (geralmente edema cerebral), infartos por embolia artério-arterial (principalmente com ulceração arterial ou na presença de placa rota/instável e falha terapêutica com anti-agregantes), e estenose intracraniana crítica. As bases para estas indicações são, entretanto, empíricas (nível 5). 


\section{MARCADORES DE INFLAMAÇÃO}

Recente diretriz (guideline) analisou a validade de marcadores inflamatórios, obtidos através de exames sangüíneos, para a previsão de eventos vasculares ${ }^{28}$. Vários fatores inflamatórios, como a proteína $C$ reativa ( $P C R$ ), interleucinas, fator de necrose tumoral, moléculas de adesão, atividade leucocitária, amilóide $A$, e outros, têm sido investigados $28-31$. Apenas a PCR, dosada pela técnica quantitativa ultra-sensível, tem alto valor preditivo.

Comentários: Não há estudos específicos de grande porte, que comprovem a validade da dosagem destes marcadores na fase aguda do AVC. O valor preditivo de complicação (progressão da trombose, novos êmbolos artério-arteriais) ou de indicação de condutas específicas (como procedimento cirúrgico ou intervencionista) na fase aguda ainda não estão estabelecidos (níveis 4 e 5).

\section{REFERÊNCIAS}

1. EUSI, European Stroke Iniciative. Recommendations for stroke manegement: update 2003. Cerebrovasc Dis 2004;17(Suppl 2):S1-S46.

2. Perry JR, Szalai JP, Norris JW, for the Canadian Stroke Consortium. Consensus against carotid surgery and screening for asymptomatic stenosis. Arch Neurol 1997; 54:25-28.

3. Nederkoorn PJ, van der Graaf Y, Hunink M. Duplex ultrasound and magnetic resonance angiography compared with digital subtraction angiography in carotid artery stenosis: a systematic review. Stroke 2003;34:1324-1332.

4. Hirai T, Korogi Y, Ono K, et al. Prospective evaluation of suspected steno-occlusive disease of the intracranial artery: combined MR angiography and CT angiography compared with digital subtraction angiography. Am J Neuroradiol 2002;23:93-101.

5. Py MO, André C, Azevedo FS, Domingues RC, Salomão RF. Internal ca rotid stenosis - comparison of duplex-scan and magnetic resonance angiography with digital subtraction angiography. Arq Neuropsiquiatr 2001;59:665-671.

6. Anderson GB, Ashforth R, Steinke DE, Ferdinandy R, Findlay JM. CT angiography for the detection and characterization of carotid artery bifurcation. Stroke 2000;31:2168-2174.

7. Wildermuth S, Knauth M, Brandt T, Winter R, Sartor K, Hacke W. Role of CT angiography in patient selection for thrombolytic therapy in acute ischemic stroke. Stroke 1998;29:935-938.

8. Verro P, Tanenbaum LN, Borden NM, Sen S, Eshkar N. CT angiography in acute ischemic stroke: preliminary results. Stroke 2002;33:276-278.

9. Sloan MA, Alexandrov AV, Tegeler CH, et al. Assessment: transcranial Doppler ultrasonography. Neurology 2004;62:1468-1481.

10. Alexandrov AV, Joseph M transcranial Doppler: an overview of its clinical applications. Internet J Emerg Intensive Care Med 2000;4:1.

11. Moore WS, Barnett HJM, Beebe HG, et al. Guidelines for carotid endarterectomy. A multidisciplinary consensus statement from the ad Hoc Committee, American Heart Association. Stroke 1995;26:188-201.

12. Johnston DCC, Chapman KM, Goldstein LB. Low rate of complications of cerebral angiography in routine clinical practice. Neurology 2001;57:2012-2014.

13. Qureshi AI, Suri FK, Ali Z, et al. Role of conventional angiography in evaluation of patients with carotid artery stenosis demonstrated by Doppler ultrasound in general practice. Stroke 2001;32:2287-2291.

14. Heiserman JE, Dean BL, Hodak JÁ, et al. Neurologic complications of cerebral angiography. Am J Neuroradiol 1994;15:1401-1407.

15. Biller J, Adams HP, Boarini D, Godersky JC, Smoker WR, Kongable G. Intraluminal clot of the carotid artery: a clinical-angiographic correlation of nine patients and literature review. Surg Neurol 1986;25:467-477.

16. Heros RC. Carotid endarterectomy in patients with intraluminal thrombus. Stroke 1988; 19:667-668.

17. Buchan A, Gates P, Petz D, Barnett HJ. Intraluminal thrombus in the cerebral circulation: implications for surgical management. Stroke 1988;19:681-687.

18. Sbarigia E, Toni D, Speziale F, et al. Emergency and early carotid endarterectomy in patients with acute ischemic stroke selected with a p redefinite protocol: a prospective pilot study. Int Angiol 2003;2: 426-430.

19. Gasecki AP, Ferguson GG, Eliasziw M, et al. Early endarterectomy for severecarotid artery stenosis after a nondisabling stroke: results from the North American Symptomatic Carotid Endarterectomy Trial. J Vasc Surg 1994;20:288-295.

20. Rothwell PM, Eliasziw M, Gutnikov AS, Warlow CP, Barnett HJM, for the Carotid Endarterectomy trialists Collaboration. Endarterectomy for symptomatic carotid stenosis in relation to clinical subgroups and timing of surgery. Lancet 2004;363:915-24.

21. Johnston SC, Gress DR, Browner WS, Sidney S. Short-term prognosis after emergency department diagnosis of TIA. JAMA 2000;284:29012906.

22. Gagliardi RJ, Raffin CN, Massaro AR, Fabio SC - Sociedade Brasileira de Doenças Cerebrovasculares. Primeiro consenso brasileiro do tratamento da fase aguda do acidente vascular cerebral. Arq Neuropsiquiatr 2001;59:972-980.

23. Raffin CN, Gagliardi RJ, Massaro AR, et al. Sociedade Brasileira de Doenças Cerebrovasculares. Primeiro consenso brasileiro para trombólise no acidente vascular cerebral isquêmico agudo. Arq Neuropsiquiatr 2002;60:675-680.

24. Progress collaborative Group. Randomised trial of perindropil-based blood-pressurelowering regimen among 6105 individuals with previous stroke or transient ischemic attack. Lancet 2001;358:1033-1041.

25. Teo K, Yusuf S, Anderson C, et al. ONTARGET/TRANSCEND Investigators. Rationale, design, and baseline characteristics of 2 large, simple, randomized trials evaluating telmisartan, ramipril, and their combination in high-risk patients: the Ongoing Telmisartan Alone and in Combination with Ramipril Global Endpoint Trial/ Telmisartan Randomized Assessment Study in ACE Intolerant Subjects with Cardiovascular Disease (ONTARGET/TRANSCEND) trials. Am Heart J 2004;148:52-61.

26. Amarenco P, Bogousslavsky J, Callahan AS, et al. SPARCL Investigators. Design and baseline characteristics of the stroke prevention by aggressive reduction in cholesterol levels (SPARCL) study. Cerebrovasc Dis 2003;16:389-395.

27. Lyrer P, Engelter S. Antith rombotic drugs for carotid artery dissection (Cochrane Review). In The Cochrane Library, 4, 2001. Oxford: Update Software.

28. Pearson TA, Mensah GA, Alexander RW, et al. Markers of inflammation and cardiovascular disease: application to clinical and public health practice: a statement for healthcare professionals from the Centers for Disease Control and Prevention of the American Heart Association. Circulation 2003;107:499-511.

29. Cao JJ, Thach C, Manolio TA, et al. C-reactive protein, carotid intimamedia thickness, and incidence of ischemic stroke in the elderly: the Cardiovascular Health Study. Circulation 2003;108:166-170.

30. Curb JD, Abbott RD, Rodriguez BL, et al. C-reactive protein and the future risk of thromboembolic stroke in healthy men. Circulation 2003;107:2016-2020

31. Blake GL, Ridker PM. Novel clinical markers of vascular wall inflammation. Cir Res 2001;89:763-771. 